\title{
The role of attainability and external focus of attention on standing long jump performance
}

\author{
ADAM KING ${ }^{1}$ | MAX POWER ${ }^{1}$ \\ 1 Texas Christian University, Fort Worth, TX, United States. \\ Correspondence to: Adam King. \\ email: a.king@tcu.edu \\ https://doi.org/10.20338/bjmb.v15i3.232
}

\begin{abstract}
HIGHLIGHTS
- An external focus of attention facilitates motor performance and learning

- Jump performance was greatest with the target placed beyond maximum distance

- Vertical and anterior-posterior impulses were greatest for the beyond maximum distance target

- Individualized targets can be used to increase

standing long jump performance
\end{abstract}

\section{ABBREVIATIONS}

ANOVA Analysis of variances

AP Anterior-posterior

FOA External focus attention

GRFimpulse Impulse of the ground reaction force

GRF $_{\text {peak }}$ Peak of the ground reaction

force

$\mathrm{n}_{\mathrm{p}}^{2} \quad$ Partial eta squared

SLJ Standing long jump

$\mathrm{T}_{\max } \quad$ Target placed at maximum distance

$T_{\max +10} \quad$ Target placed at maximum distance plus $10 \%$

$\mathrm{T}_{\mathrm{no}} \quad$ Without a visual target

$\mathrm{T}_{\text {perceived }} \quad$ Perceived maximum jump

Distance
BACKGROUND: External focus of attention (FOA) promotes enhanced performance and learning in comparison to internal FOA; however, several dimensions associated with external FOA appear to have varying influence on motor skill performance.

AIM: The purpose of this study was to investigate whether an attainability aspect of external FOA elicits enhanced standing long jump performance.

METHOD: Fifteen healthy males performed standing long jumps in the following conditions: without a visual target $\left(T_{n o}\right)$, target placed at maximum distance $\left(T_{\max }\right)$, and a target placed $10 \%$ beyond maximum distance $\left(T_{\max +10)}\right.$. Jump distances were measured, and kinetic data were recorded using a force platform.

RESULTS: One-way ANOVA showed significantly greater jump distances for $T_{\max +10}$ compared to $T_{\text {no }}$ and $T_{\max }$ ( $p$ $<0.01)$. Additionally, the impulse of the ground reaction force $\left(G R F_{\text {impulse }}\right)$ in the anterior-posterior direction was significantly greater for $T_{\max }$ and $T_{\max +10}$ compared to $T_{n o}(p<0.05)$.

CONCLUSION: Thus, an external FOA tailored to individual ability benefits standing long jump performance. Overall, the findings support the approach of adopting an external FOA with an emphasis on targets that are specific to the individual and based on attainability.

KEYWORDS: Attentional focus | Attainability | Kinetics | Motor performance

\section{INTRODUCTION}

When performing motor actions, individuals exhibit the ability to alter attentional focus to a variety of movement details or environmental factors. In coaching, one approach for enhancing skill acquisition and performance includes the use of varying focus of attention (FOA) on either key aspects of the movement (joint angles and ground reaction force) or consequences of the intended movement production. An abundance of evidence has shown positive performance benefits when attention is directed toward movement effects (i.e., external FOA), whereas conscious control of body movements through an internal FOA results in reduced performance levels. ${ }^{1,2}$ Additionally, external FOA promotes

\begin{tabular}{l|l|l|l|lr}
\hline King, Power & 2021 & VOL.15 & N.3 & https://doi.org/10.20338/bjmb.v15i3.232
\end{tabular}


efficient movement pattern ${ }^{3}$ as well as automaticity ${ }^{4}$ in movement control; however, it remains unclear whether there is an optimal distance of external FOA that maximizes performance.

Supporting evidence for adoption of external FOA can be found in a wide range of motor tasks. For example, while balancing on a stabilometer smaller balance errors and faster reaction times were found with attention focused externally toward markers on the platform as compared to internally. ${ }^{4}$ Additionally, the presence, rather than the absence (i.e., no overhead goal), of a suspended ball positioned directly overhead (external focus) led to significantly higher vertical jump heights for both men and women. ${ }^{5}$ Increased jump distances were also found in a standing long jump (SLJ) task when focusing on an external visual target rather than on rapid knee extension. ${ }^{6,7}$ Collectively, an external FOA promotes enhanced task outcomes for both balance and high-power jumping activities.

One dimension of external FOA that has received recent investigation relates to the manipulation of near (proximal) versus far (distal) external foci and is known as the distance effect.2,8-10 McKay and Wulf9 demonstrated the distance effect in a dart throwing task whereby novice individuals obtained improved accuracy when focusing on a distal target rather than a proximal focus of the flight of the dart. Similarly, Bell and Hardy ${ }^{11}$ found enhanced shot accuracy among skilled golfers when adopting a distal (intended shot direction) versus proximal (clubface square at impact) external focus of attention. In a standing long jump, Porter and colleagues ${ }^{10}$ showed improved performance with a far (toward a target) versus a near (next to the body) external FOA. This distal FOA benefit has also been replicated in athletic populations ${ }^{12}$ and with varying distal FOA, ${ }^{13}$ and appears to influence the performance of both novice and skilled individuals. ${ }^{14}$

Collectively, previous evidence suggests that a greater distance of an external FOA benefits motor performance; however, it remains unclear whether there is an optimal distance effect for external FOA. Additionally, the role of attainability ${ }^{15}$ has not been fully incorporated into the manipulation of external foci. While certain motor tasks, like the tennis serve and golf putt, provide a clear attainable distal FOA related to the task outcome, other tasks possess bounded performance characteristics that limit the potential influence of the distance effect. Specifically, the execution of a golf putt or tennis serve with a distal focus can be accomplished through increased force production which is practically relevant for achieving the task outcome (ball in the hole or landing in proper serving area). However, for tasks like the standing long jump a far target FOA (i.e., $3 \mathrm{~m}$ ) that is beyond the capabilities of the individual may render limited performance enhancements. This potential performance detriment of a far, distal FOA may be related to a lack of perceived attainability, diminished motivation or self-efficacy of task completion.

Coker ${ }^{15}$ explored the aspect of the distance effect by addressing the specificity of a distal FOA and found enhanced SLJ performance for a near target (pre-determined maximum jumping distance) as compared to far (3-meter target) and control ("do your best" strategy) conditions. This finding suggests that individually tailored targets versus arbitrary (i.e., 3 or $5 \mathrm{~m}$ ) external distances may be beneficial to performance ${ }^{13}$ and invites exploration around identifying a potential optimal distance effect. In support of this notion, McNevin et al. ${ }^{1}$ proposed that proximal external foci may yield similar disruptions to performance as internal FOA. Therefore, we sought to examine a fine-grain manipulation of an external FOA by incorporating the component of attainability to determine whether this distance effect influences standing long jump performance. 
An attainable target can be considered one in which the individual is physically capable of achieving success. The improved performance found by Coker ${ }^{15}$ when using an attainable target may also be related to perception capabilities. Conditions that the individual perceives as attainable appear to produce optimal movement patterns; however, perceived attainability may differ from actual task attainability. Specifically, individuals tend to underestimate the ability to successfully pass through various doorway widths. ${ }^{16}$ Despite actual pass-through ability of certain narrow doorways, most individuals deemed the doorway widths as unattainable. Task constraints can influence the perception of action boundaries (i.e., physical limitations). For example, individuals overestimated jumpable gap width distances when wearing ankle weights; however, the additional mass failed to alter the perception of unattainable (un-jumpable) gap widths. ${ }^{17}$ This finding suggests that individuals may perceive tasks to be more or less difficult depending on the relation between environmental factors and their own action capabilities. While these previous studies focused the role of attainable and unattainable conditions on perception of success, the effects of attainability on physical performance have not been fully examined.

The current study investigated whether an individually tailored external FOA elicits enhanced standing long jump performance compared to a control (i.e., no target) condition. According to Coker ${ }^{15}$ individualized jump distances within one's motor ability may be more beneficial to task performance than the use of a far (i.e., 3 meter) FOA. ${ }^{18}$ However, the manipulation of an external FOA within and slightly beyond one's measured limits is unclear. To better understand the effect of distance, the targets used in this study were placed at previously measured maximum jump distance and at a distance $10 \%$ beyond the maximum jump distance. We hypothesized that both target conditions would result in greater jump distances compared to the control (i.e., no target) condition. Additionally, we explored whether a kinetic property (e.g., ground reaction force) of standing long jump performance was influenced by such manipulations. Similar to evidence found for the vertical jump task, ${ }^{19}$ we expected that both target conditions would result in increased force production (peak and impulse) compared to the control condition.

\section{MATERIAL AND METHODS}

\section{Participants}

Fifteen physically active males (age: $20.5 \pm 0.92$ years, height: $180 \pm 4.88 \mathrm{~cm}$, weight: $79.5 \pm 10.4 \mathrm{~kg}$ ) were recruited to participate in the study. All participants exercised at least three times a week for a total of 150 minutes over the past three months prior to testing and lacked formal training in the SLJ. Participants reported no lower extremity injuries within the past year and no lower extremity injuries requiring surgery for at least three years prior. Research approval was granted by the Institutional Review Board and written informed consent was obtained from each participant prior to testing.

Task

After obtaining consent, each participant performed a warm-up consisting of 10 squats, 10 jumping jacks, 20 lunges (10 per leg), and 10 squat jumps with minimal rest between exercises. Then, the participant watched a one-minute video describing the basic mechanics of the SLJ. Following the video, five practice jumps from the force plate were allowed. Both the jumping and landing surfaces were firm and flat. 
Prior to the execution of the standing long jump task, the participant stood at the start line marked on top of the force plate and used a laser pointer to identify perceived maximum jump distance ( $\left.T_{\text {perceived }}\right)$. The first four trials of data collection were performed without any special instructions or visual target in order to determine the maximum jump distance $\left(T_{\max }\right)$ of the participant with the furthest jump recorded. In random order, the participant performed three trials for each of the following conditions: target located at maximum jump distance ( $\left.T_{\max }\right)$, target located at maximum distance plus 10\% ( $\left.T_{\max +10}\right)$, and without a visual target $\left(T_{n o}\right)$. Target distances $\left(T_{\max }\right.$ and $\left.T_{\max +10}\right)$ were marked with a red laser pointing perpendicular to the line of sight of the participant. Participants were instructed to jump as far as possible for each trial. A successful landing was defined as sticking the initial landing location with both feet (landing without shuffling or shifting feet). Jump distances were measured with a standard measuring tape and recorded from the toes at takeoff to the heels at landing. Between each trial, the participant rested while standing or slowly walking for two minutes. Kinetic data were collected using a force plate (AMTI OR6-7) with peak (GRF peak) and integrated ground reaction force (GRF impulse) in anterior-posterior (AP) and vertical directions computed for each jump.

\section{Statistical Analysis}

A paired t-test was used to determine whether individuals' perceived jump distances differed from their initial maximal jump performance. Separate one-way analysis of variances (ANOVA) were performed using the Statistical Package for Social Sciences (IBM Corp.) to analyze jump distances as well as ground reaction forces between conditions. For post-hoc tests, Bonferroni adjustments were used when appropriate. Partial eta squared $n_{p}^{2}$ was used to determine effect size with magnitudes classified as trivial $(0-$ $0.009)$, small $(0.01-0.059)$, medium $(0.060-0.139)$, or large $\left(0.140\right.$ and greater). ${ }^{20}$ All significance levels were set at $p<0.05$.

\section{RESULTS}

No significant difference was found between $T_{\text {perceived }}$ and $T_{\max }$ jump distances, $p=$ 0.64 , with participants displaying a tendency to overestimate actual jump distances ( $T_{\text {perceived: }} M=221 \mathrm{~cm}, S D=32.03 \mathrm{~cm}$; $T_{\max }: M=218 \mathrm{~cm}, S D=29.91 \mathrm{~cm}$ ). We quantified the difference between $T_{\text {perceived }}$ and $T_{\max }$ separately for individuals that overestimated $(n=10)$ and underestimated $(n=7)$ jump distance and found that overestimations $(M=21$ $\mathrm{cm}, S D=10.19 \mathrm{~cm})$ tended to be smaller than underestimation $(M=33 \mathrm{~cm}, S D=18.58$ $\mathrm{cm})$.

A significant main effect of condition was found for jump distances, $F(2,34)=$ $15.80, p<0.001, n_{p}^{2}=0.48$. Post hoc comparisons revealed that $T_{\max +10}(M=226.22 \mathrm{~cm}$, $S D=27.94 \mathrm{~cm})$ jump distances were significantly greater than $T_{\max }(M=222.50 \mathrm{~cm}, S D=$ $27.18 \mathrm{~cm})$ and $\mathrm{T}_{\text {no }}(M=219.22 \mathrm{~cm}, S D=28.44), p<0.01$ (Figure 1). The jump distances between $T_{\max }$ and $T_{n o}$ trended towards a significance difference, $p=0.06$. 


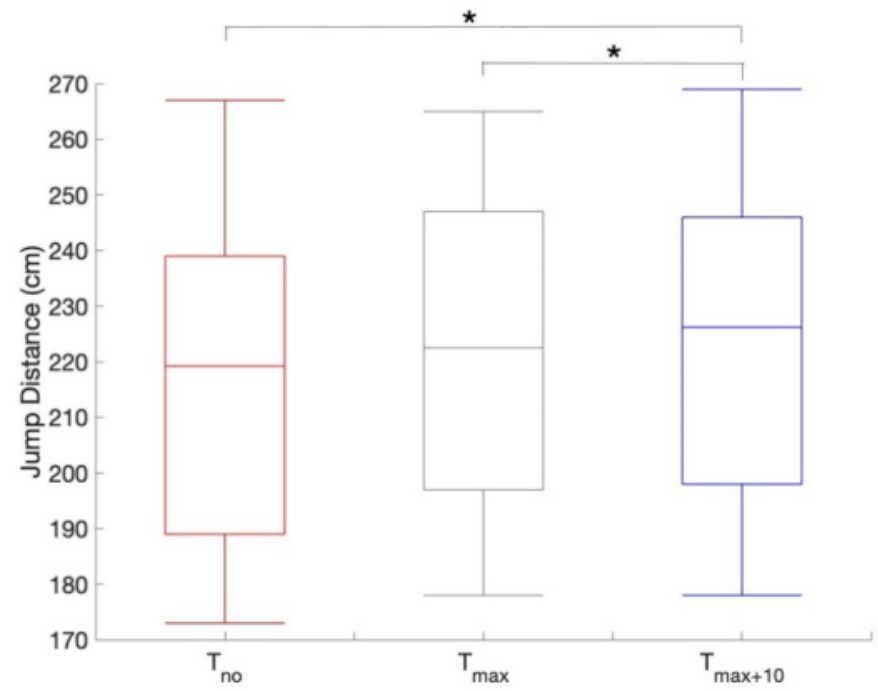

Figure 1. Average Jump Distances. Jump distances in the $T_{n o}, T_{\max }$, and $T_{\max +10}$ conditions. $T_{\max +10}$ was significantly farther than both the $T_{\text {no }}$ and $T_{\max }$ conditions $(p<0.01)$.

Table 1 displays the kinetic data in the anterior-posterior (AP) and vertical directions for the three jump conditions. The results of the repeated measures ANOVA revealed a significant effect of condition for GRFimpulse, $F(2,28)=8.37, p<0.01, n_{p}^{2}=0.37$ in the AP direction. The AP GRF impulse for both $T_{\max }(p<0.05)$ and $T_{\max +10}(p<0.001)$ were significantly larger than $\mathrm{T}_{\text {no }}$ (see Table 1). The $\mathrm{GRF}_{\text {peak }}$ in the AP direction trended towards a significance effect of condition, $F(2,28)=3.09, p=0.06, n_{p}^{2}=0.18$. In the vertical direction, no significant differences were found for GRFimpulse, $F(2,28)=1.86, p=0.18$, $n_{p}^{2}=0.18$ and $\mathrm{GRF}_{\text {peak }}, F(2,28)=0.55, p=0.58, n_{p}^{2}=0.04$.

Table 1 - Mean and Standard Deviation for Ground Reaction Force data.

\begin{tabular}{|c|c|c|c|c|}
\hline & \multicolumn{2}{|c|}{ Anterior-Posterior } & \multicolumn{2}{|c|}{ Vertical } \\
\hline & Impulse (Ns) & Peak (N) & Impulse (Ns) & Peak (N) \\
\hline $\mathrm{T}_{\mathrm{no}}$ & $224.58 \pm 44.48$ & $759.83 \pm 177.78$ & $1053.44 \pm 197.69$ & $1619.40 \pm 243.72$ \\
\hline$T_{\max }$ & $228.31 \pm 44.44^{*}$ & $773.09 \pm 174.34$ & $1089.16 \pm 202.60$ & $1638.14 \pm 250.13$ \\
\hline$T_{\max +10}$ & $231.02 \pm 45.25^{\star}$ & $780.24 \pm 190.15$ & $1113.00 \pm 198.68$ & $1623.39 \pm 272.68$ \\
\hline
\end{tabular}

The purpose of this study was to examine whether an individually tailored external FOA enhanced standing long jump (SLJ) performance. In an extension of previous investigations demonstrating the benefits of external FOA on motor performance, $, 6,7,15,18$ we sought to determine if a fine-grain manipulation of an external target (i.e., distance 
effect) increased jump distances. Consistent with the attainability perspective proposed by Coker, ${ }^{15}$ we hypothesized that both external targets $\left(T_{\max }\right.$ and $\left.T_{\max +10}\right)$ would result in greater jump distances than a control $\left(T_{n o}\right)$ condition with $T_{\max +10}$ resulting in the farthest jump distances. The findings supported this predication showing that SLJ performance scaled according to the three conditions ( $T_{n o}, T_{\max }$, and $\left.T_{\max }+10\right)$.

The results of this study support the notion that an external FOA allows enhanced performance outcomes as compared to a control condition. ${ }^{4}$ The use of a visual target led to increased jump distances as compared to no target. In all conditions, the instructions emphasized jumping as far as possible; however, during the $T_{\text {no }}$ condition jump distances were the least suggesting that this control state did not facilitate an optimal coordination pattern for the SLJ movement. Similarly, Coker ${ }^{15}$ found this phenomenon in that when individuals were instructed to "do your best", maximal performance levels were not achieved. Therefore, intentionally directing attention externally appears to aid individuals in achieving superior movement performances.

The external FOA used in this study was clearly discernible from an approach with a proximal or internal focus; however, the physical distance between the $T_{\max }$ and $T_{\max +10}$ targets may not have been distinguishable by individuals when executing the standing long jumps. According to McNevin et al.., ${ }^{1}$ individuals clearly discriminate body movement cues from distal distance effects; but, this may not be the case when adopting a proximal distance focus. Currently, this perspective has not been fully explored to provide clear practical guidance. Here, the small physical difference between the two targets may have prompted the group of unskilled individuals to use the same information resulting in similar movement solutions that produced similar results. According to the constrained action hypothesis, external foci facilitate enhanced movement automaticity and efficiency;2,3 however, whether our distant effect manipulation influences the self-organization component requires further investigation. Nonetheless, the far target increased jump distance independent of whether individuals perceived the physical distance difference and future investigations should explore how this manipulation impacts movement control.

From the kinetic data, the findings showed that individuals generated greater force (peak and impulse) for both target conditions which may be related to a more efficient muscle fiber recruitment pattern used to achieve enhanced task performance outcome. This results is consistent with previous evidence ${ }^{15}$ showing that attainable targets outperformed a fixed distance far condition (3 meters). Thus, an external FOA positioned beyond a person's capabilities may negatively influence state self-confidence. State selfconfidence describes a person's perception of ability to complete a task at a particular moment and directly affects behavioral responses. ${ }^{21}$ Therefore, the enhanced jump distances with the far target in this study may be related to a positive perception of ability, and certain target distances may not enhance jumping performance. Future studies should investigate whether an optimal distance relative to individual ability leads to maximized performance in the SLJ task.

Typically, investigations of external FOA have used target distances that are either partially or completely standardized across all participants; 15,18 however, it is important to note that each jumper likely exhibits different motor abilities as well as perceptions of ability. Consistent with this perspective, the results revealed a high degree of individual variability (i.e., a wide range of over- and under-estimations) with a general overestimation of jump ability across all individuals suggesting a disparity between perceived and actual 
attainability. Thus, using a common far target (i.e., 3 meters) may not appropriately challenge individuals with different motor abilities. The tailored target distance based on predetermined maximum jumps significantly improved jump performance, suggesting that the target may have allowed for an improved organization of movements that led to the observed performance enhancement. ${ }^{4}$

Previous investigations have shown that a variety of biomechanical properties, such as joint (knee, ankle, and hip) angles at takeoff, projection angle and center of gravity velocity contribute to SLJ performance. ${ }^{6,22,23}$ Furthermore, in addition to changes of performance outcome the manipulation of focus of attention influences movements properties (i.e., kinematics, kinetics, etc.). Our findings showed that select kinetic variables scaled with respect to the different conditions. Specifically, AP and vertical GRF impulses increased from the control to the $T_{\max }$ to the $T_{\max +10}$ conditions as did the peak AP GRF. Using an external FOA, Wulf and Dufek ${ }^{24}$ found similar evidence of greater impulses during a vertical jump task that was followed by a demonstration of lower muscle activity with improved performance outcomes (i.e., greater jump heights). ${ }^{24}$ The current findings are consistent with such findings showing that external FOA improves performance and facilitates the production of effective and efficient movement patterns. Future examinations of the lower extremity neuromuscular activity under the current manipulations would provide additional insight related to changes in movement organization of the SLJ task.

\section{CONCLUSION}

In summary, the current study supports the perspective that an external FOA leads to improved SLJ performance compared to a lack of attentional focus and that extending such FOA slightly beyond one's maximum performance offers additional task outcome benefits. Furthermore, the results are consistent with existing evidence that individually tailored target enhanced SLJ jump distances. Further investigation is needed to better understand the extent of the target distance from the individual needed to maximize improved performance.

\section{REFERENCES}

1. McNevin $\mathrm{NH}$, Shea $\mathrm{CH}$, Wulf $\mathrm{G}$. Increasing the distance of an external focus of attention enhances learning. Psychol Res. 2003;67(1):22-9.

2. Kal EC, Van Der Kamp J, Houdijk H. External attentional focus enhances movement automatization: A comprehensive test of the constrained action hypothesis. Hum Mov Sci. 2013;32(4):527-39.

3. Vance J, Wulf G, Töllner T, McNevin N, Mercer J. EMG activity as a function of the performer's focus of attention. J Mot Behav. 2004;36(4):450-9.

4. Wulf $\mathrm{G}, \mathrm{McNevin} \mathrm{N}$, Shea $\mathrm{CH}$. The automaticity of complex motor skill learning as a function of attentional focus. Q J Exp Psychol Sect A Hum Exp Psychol. 2001;54(4):114354. 10.1080/713756012 
5. Ford KR, Myer GD, Smith RL, Byrnes RN, Dopirak SE, Hewett TE. Use of an overhead goal alters vertical jump performance and biomechanics. J Strength Cond Res. 2005;19(2):394-9. 10.1519/15834.1

6. Ducharme SW, Wu WFW, Lim K, Porter JM, Geraldo F. Standing Long Jump Performance With an External Focus of Attention Is Improved as a Result of a More Effective Projection Angle. J Strength Cond Res. 2016;30(1):276-81. 10.1519/JSC.0000000000001050

7. Vidal A, Wu W, Nakajima M, Becker J. Investigating the Constrained Action Hypothesis: A Movement Coordination and Coordination Variability Approach. J Mot Behav. 2017;2895(September):1-10. 10.1080/00222895.2017.1371111

8. Kearney PE. A distal focus of attention leads to superior performance on a golf putting task. Int J Sport Exerc Psychol. 2015;13(4):371-81. 10.1080/1612197X.2014.993682

9. McKay B, Wulf $\mathrm{G}$. A distal external focus enhances novice dart throwing performance. Int $J$ Sport Exerc Psychol. 2012;10(2):149-56.

10. Porter JM, Anton PM, Wu WFW. Increasing the distance of an external focus of attention enhances standing long jump performance. J Strength Cond Res. 2012;26(9):2389-93.

11. Bell JJ, Hardy J. Effects of Attentional Focus on Skilled Performance in Golf. J App/ Sport Psychol. 2009;21(2):163-77. 10.1080/10413200902795323

12. Porter JM, Anton PM, Wikoff NM, Ostrowski JB. Instructing skilled athletes to focus their attention externally at greater distances enhances jumping performance. J Strength Cond Res. 2013;27(8):2073-8.

13. Westphal W, Porter JM. Increasing the Distance of an External Focus of Attention has Limited Effects on Standing Long Jump Performance. Int J Exerc Sci. 2013;6(4):300-309.

14. Singh $H$, Wulf $G$. The distance effect and level of expertise: Is the optimal external focus different for low-skilled and high-skilled performers? Hum Mov Sci. 2020;73(February).

15. Coker C. Optimizing External Focus of Attention Instructions: The Role of Attainability. J Mot Learn Dev. 2016;4(1):116-25.

16. Davis TJ, Riley MA, Shockley K, Cummins-Sebree S. Perceiving affordances for joint actions. Perception. 2010;39(12):1624-44.

17. Lessard DA, Linkenauger SA, Proffitt DR. Look before you leap: Jumping ability affects distance perception. Perception. 2009;38(12):1863-6.

18. Porter JM, Ostrowski EJ, Nolan RP, Wu WFW. Standing long-jump performance is enhanced when using an external focus of attention. J Strength Cond Res. 2010;24(7):1746-50.

19. Wulf G, Dufek J. Increased jump height with an external focus due to enhanced lower extremity joint kinetics. J Mot Behav. 2009;41(5):401-9.

20. Cohen J. A power primer. Psychol Bull. 1992;112:155-9.

21. Vealey RS. Conceptualization of Sport-Confidence and Competitive Orientation: Preliminary Investigation and Instrument Development. J Sport Psychol. 2016;8(3):221-46.

22. Wakai M, Linthorne NP. Optimum take-off angle in the standing long jump. Hum Mov Sci. 2005;24(1):81-96. 
23. Aguado X, Izquierdo M, Montesinos JL. Kinematic and kinetic factors related to the standing long jump performance. J Hum Mov Stud. 1997;32(4):156-69.

24. Wulf G, Dufek JS, Lozano L, Pettigrew C. Increased jump height and reduced EMG activity with an external focus. Hum Mov Sci. 2010;29(3):440-8.

\section{ACKNOWLEDGEMENTS}

The authors would like to thank Joel Petri and Curtis Hanson for their contributions to data collections.

Citation: King A, Power M. (2021). The Role of Attainability and External Focus of Attention on Standing Long Jump Performance. Brazilian Journal of Motor Behavior, 15(3): 207-215.

Editors: Dr Fabio Augusto Barbieri - São Paulo State University (UNESP), Bauru, SP, Brazil; Dr José Angelo Barela São Paulo State University (UNESP), Rio Claro, SP, Brazil; Dr Natalia Madalena Rinaldi - Federal University of Espírito Santo (UFES), Vitória, ES, Brazil.

Copyright:@ 2021 King and Power and BJMB. This is an open-access article distributed under the terms of the Creative Commons Attribution-Non Commercial-No Derivatives 4.0 International License which permits unrestricted use, distribution, and reproduction in any medium, provided the original author and source are credited. Funding: This research did not receive any specific grant from funding agencies in the public, commercial, or not-forprofit sectors.

Competing interests: The authors have declared that no competing interests exist.

DOI: https://doi.org/10.20338/bjmb.v15i3.232 